\title{
Fusiform Cervical Mass in a 6-Year Old Boy; Do not Forget the Thymic Cyst
}

\author{
Ioannis Patoulias ${ }^{1}$, Magdalini Mitroudi ${ }^{1}$, Vasilios Rafailidis ${ }^{2}$, Thomas Feidantsis ${ }^{1}$, \\ Constantine Theocharides ${ }^{3}$, Parthena Kampouridou 4 , Dimitrios Patoulias ${ }^{5, *}$
}

\begin{abstract}
Cervical thymic cyst is a rare clinical entity, with approximately one hundred cases reported in the literature so far. The purpose of this case report is to highlight some certain features, along with an extensive research of the relevant literature.

A 6-year-old boy was admitted to the Otorhinolaryngology Department due to the presence of a left-sided, painless, latero-cervical swelling, first observed by his parents 2 weeks ago. Physical examination revealed a painless, well-delineated mass, with no signs of inflammation. No enlarged cervical nodes were present. The mass extended from the mandibular angle, under the sternocleidomastoid muscle, in proximity with the ipsilateral neurovascular bundle. Ultrasound transverse gray-scale panoramic view detected a wellcircumscribed lesion, with fine echogenic foci, appearing in close proximity with the upper pole of the left thyroid lobe and the ipsilateral common carotid artery. Elective surgical intervention with complete mass excision was performed. Histopathological examination confirmed the diagnosis of a cervical thymic cyst.

Cervical thymic remnants represent a group of neck masses that pediatricians and pediatric surgeons should consider in differential diagnosis of both cystic and solid neck masses. Most cystic cervical thymic masses are found in the lower third of the anterior neck, extending deep to the sternocleidomastoid muscle, featuring close anatomic relationship with the composites of the ipsilateral carotid sheath. Elective surgery is kept as the treatment of choice, after ruling out the possibility of subject immunologic disturbance.
\end{abstract}

\section{KEYWORDS}

thymic cyst; cervical thymic remnant; child; Hassall's corpuscles; thymo-pharyngeal duct

\section{AUTHOR AFFILIATIONS}

${ }^{1}$ First Department of Pediatric Surgery, Aristotle University of Thessaloniki, General Hospital "G. Gennimatas", Thessaloniki, Greece

${ }^{2}$ Department of Radiology, Aristotle University of Thessaloniki, General Hospital "AHEPA", Thessaloniki, Greece

${ }^{3}$ Department of Pathology, General Hospital "G. Gennimatas", Thessaloniki, Greece

${ }^{4}$ Department of Pediatrics, General Hospital "G. Gennimatas", Thessaloniki, Greece

${ }^{5}$ First Department of Internal Medicine, General Hospital "Hippokration", Thessaloniki, Greece

*Corresponding author: Konstantinoupoleos 49, 54642, Thessaloniki, Greece; e-mail: dipatoulias@gmail.com

Received: 25 March 2019

Accepted: 21 June 2019

Published online: 30 October 2019

Acta Medica (Hradec Králové) 2019; 62(3): 117-122

https://doi.org/10.14712/18059694.2019.135

(c) 2019 The Authors. This is an open-access article distributed under the terms of the Creative Commons Attribution License (http://creativecommons.org/licenses/by/4.0), which permits unrestricted use, distribution, and reproduction in any medium, provided the original author and source are credited. 


\section{INTRODUCTION}

Thymus arises from the third and fourth pharyngeal pouches during the sixth gestational week $(1,2)$. Over the next few weeks, thymic tissue migrates caudally and medially along the thymo-pharyngeal duct to the superior and anterior mediastinum $(1,2)$. During childhood and adolescence, it features generally soft-tissue attenuation and undergoes fibro-fatty involution with aging $(1,2)$.

Thymus is organized into multiple lobules, arranged into an outer cortex and an inner medulla. The cortex is composed of immature T-lymphocytes and thymic epithelial cells; the medulla contains maturing lymphocytes and whorls of spindle-shaped epithelial cells, which create Hassall corpuscles with keratinized cores (3). Thymus is usually located in the anterior mediastinum; however, ectopic thymic tissue can be found anywhere, from the skull base to the diaphragm, along the thymo-pharyngeal duct $(4,5)$.

Cervical thymic cysts are rare and usually present during the first decade of life $(6,7)$. Heise et al have previously demonstrated that cervical thymic cysts constitute almost $0.3 \%$ of all congenital cervical cysts found in childhood (8). Cervical thymic cyst represents a rare entity, with only about one hundred cases reported in the literature so far (9). According to findings from patients who underwent neck or thyroid ultrasonography, the prevalence of ectopic thymus varies widely from 2.3 to $17.3 \%(10,11,12)$. Kim et al examined 3,195 patients, demonstrating a prevalence equal to $0.4 \%$ (13). Hsieh et al. demonstrated that among 331 patients under the age of 18 years presenting with cystic neck masses 181 (54.68\%) had thyroglossal cysts, 83 had cystic hygromas (25.08\%), 54 had branchial cleft cysts $(16.31 \%), 3$ had bronchogenic cysts $(0.91 \%)$, while 9 cases $(2.72 \%)$ remained unclassified. Interestingly, only one case was diagnosed as thymic cyst (0.30\%) (8).

Despite the fact that the majority of ectopic thymic lesions are located in proximity with the thyroid gland, many pediatricians are not familiar with these entities (10). Through this case study, we aim at highlighting some certain features of thymic cysts, along with a comprehensive research of the relevant literature.

\section{CASE DESCRIPTION}

A 6-year-old boy was admitted to the Otorhinolaryngology Department due to the presence of a left, painless, latero-cervical, fusiform mass, which was first observed by his parents 2 weeks ago. Mass volume did not alter, while it did not exert pressure phenomena on the adjacent anatomic structures. After a detailed medical history, it was reported that the patient had recurrent episodes of inspiratory stridor without respiratory distress, lasting a few minutes. Physical examination revealed the presence of a painless, well-delineated, soft mass, while no signs of inflammation or enlarged cervical lymph nodes were detected. The mass extended from the mandibular angle, under the sternocleidomastoid muscle, in proximity with the ipsilateral neurovascular bundle. Rest physical examination was unremarkable. Performance of the Valsalva maneuver was not associated with a significant alteration in mass dimensions. Chest radiograph did not reveal any co-existing pathology.

Performance of transverse gray-scale panoramic view ultrasound showed the cystic lesion located on the left lateral neck region (Figure 1).

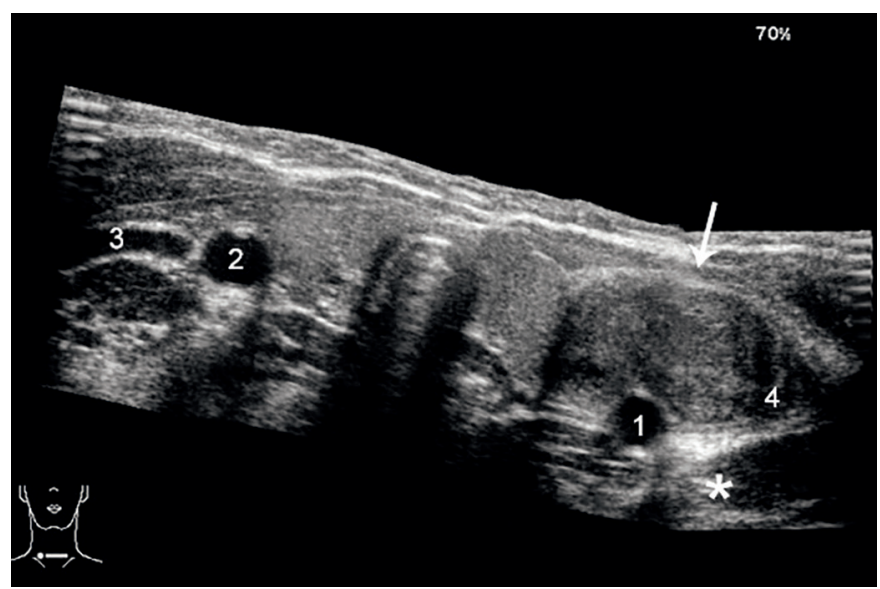

Fig. 1 Ultrasound transverse gray-scale panoramic view showing the large cystic lesion situated on the left lateral neck region. The lesion (arrow) is well-circumscribed, contains fine echogenic foci and appears in close proximity with the left thyroid lobe and the common carotid artery. On pressure by the probe, the echogenic foci could be seen moving. Posterior acoustic enhancement (asterisk) suggests the cystic nature of the lesion. 1: left common carotid artery, 2: right common carotid artery, 3: right jugular vein, 4: displaced left internal jugular vein.

The lesion was well-circumscribed, containing fine echogenic foci and appearing in close proximity with the left thyroid lobe and the common carotid artery. On pressure by the probe, the echogenic foci could be seen moving (Figure 2).

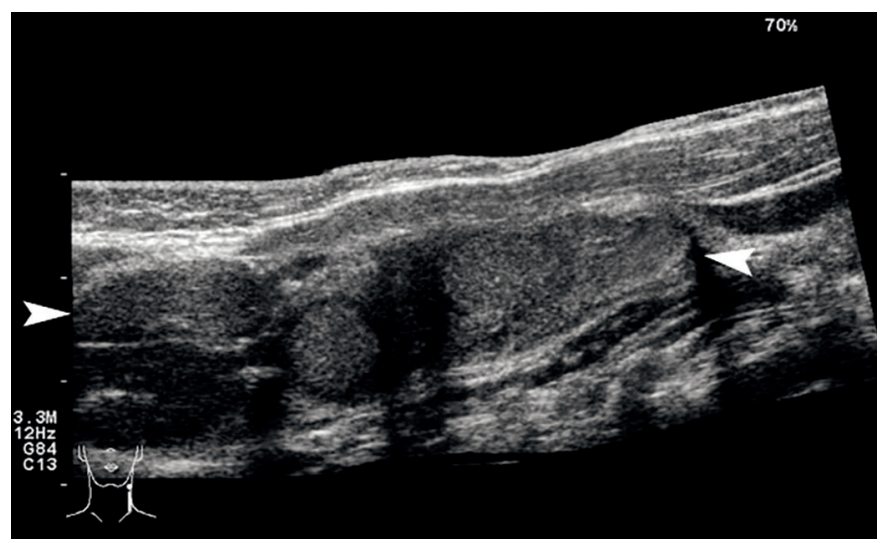

Fig. 2 Ultrasound longitudinal gray-scale panoramic view showing the elongated cystic lesion (highlighted by the arrowheads). The multi-locular nature of the lesion, containing septations and the posterior acoustic enhancement can be readily appreciated in this image.

The multi-locular nature of the lesion, containing septations, was also documented. Color Doppler transverse view revealed the absence of vascularity within the lesion, along with the displacement of the internal jugular vein and the common carotid artery (Figure 3 ). 


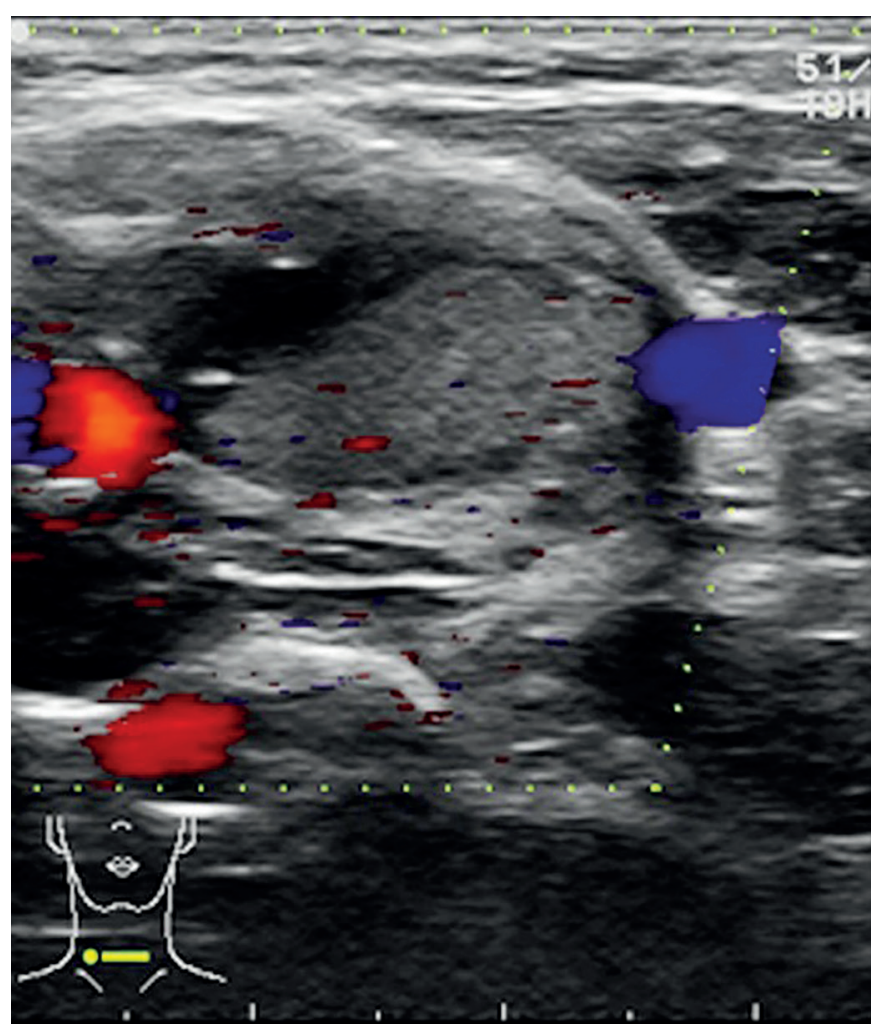

Fig. 3 Color Doppler transverse view showing the absence of vascularity within the lesion, the displacement of the jugular vein (blue color) and the common carotid artery (red color). Note is made of a fluid-fluid level formed between an anechoic and an echogenic content.

We also performed a chest CT scan, which confirmed the normal configuration of the 2 lobes of the thymus gland. Laboratory exams, including thyroid function tests, were unremarkable.

Thus, we proceeded with the elective surgical intervention. Elective surgical excision was performed under general endotracheal anesthesia. A curved skin incision along the anterior surface of the mass was performed. A multi-cystic mass, extending between the left gonial angle of the mandible and the upper pole of the ipsilateral thyroid lobe was bluntly dissected and completely removed (Figures 4,5 ).

Meticulous mass dissection from the left internal jugular vein and the left common carotid artery (carotid sheath) was performed. No extension of the mass to the mediastinum was documented (Figure 6).

Macroscopically, the surgical specimen consisted of a solid-cystic mass, which appeared multilocular, sizing $14.5 \times 2.5 \times 2.9 \mathrm{~cm}$ in dimensions (Figure 7).

Applied drain on the surgical site was removed 48 hours postoperatively and the patient was discharged home on the third postoperative day in excellent general condition.

Histopathological examination revealed that the solid part of the lesion consisted of thymic tissue with Hassall's corpuscles. In proximity to its solid part, a fibrous wall lined with stratified epithelium was also identified. Additionally, lymphocytic infiltration of the cystic wall and several cholesterol granulomas were found. Crystals

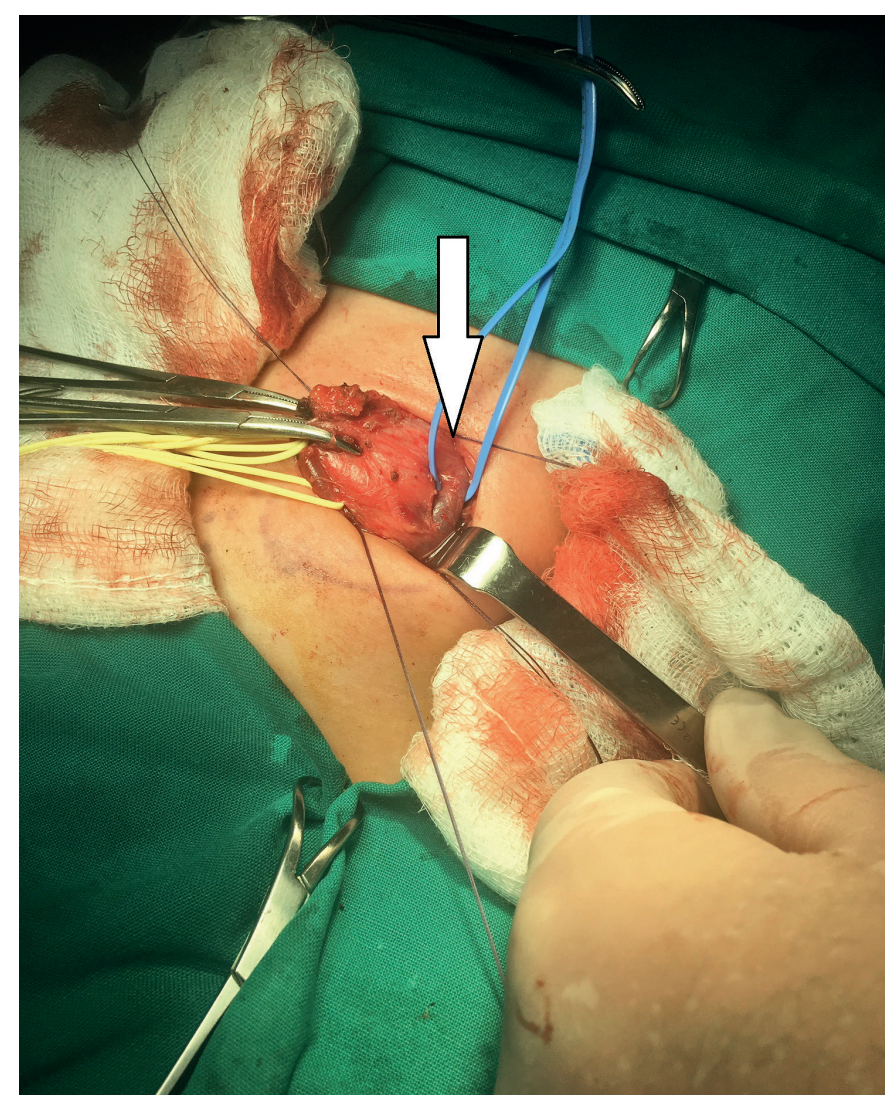

Fig. 4 The mass was bluntly dissected and completely removed, without being opened. Notice the close anatomic relationship with the internal jugular vein (white arrow).

of cholesterol crystals were also present in the luminal fluid (Figures 8, 9).

Postoperative course was uneventful, while the patient remains asymptomatic, with no symptoms of recurrence during the follow up. The latter constituted of an ultrasonographic examination performed in the 6 and 12 months postoperatively, with no radiologic signs of thymic cyst's recurrence, along with meticulous physical examination.

\section{DISCUSSION}

Differential diagnosis of neck masses during childhood includes thyroglossal duct cyst, cystic hygroma, bronchogenic cyst, branchial cyst, cervical lymphadenopathy with cystic degeneration, laryngocele, tumors and dermoid cyst. Rarely, it may also include a thyroid or parathyroid cyst $(7,14)$.

The increasing number of cervical thymic cysts reported in the literature during the last few years reflects probably the greater awareness of pediatricians and pediatric surgeons concerning this rare clinical entity. It cannot be excluded that in the past lots of cases of thymic cyst might have been misdiagnosed as brachial cleft cysts. Due to the gradual development of atrophy of the thymic remnants, tissue sampling from several different parts of the surgical specimen may be required, in order to be documented the right diagnosis of a thymic cyst (15). 


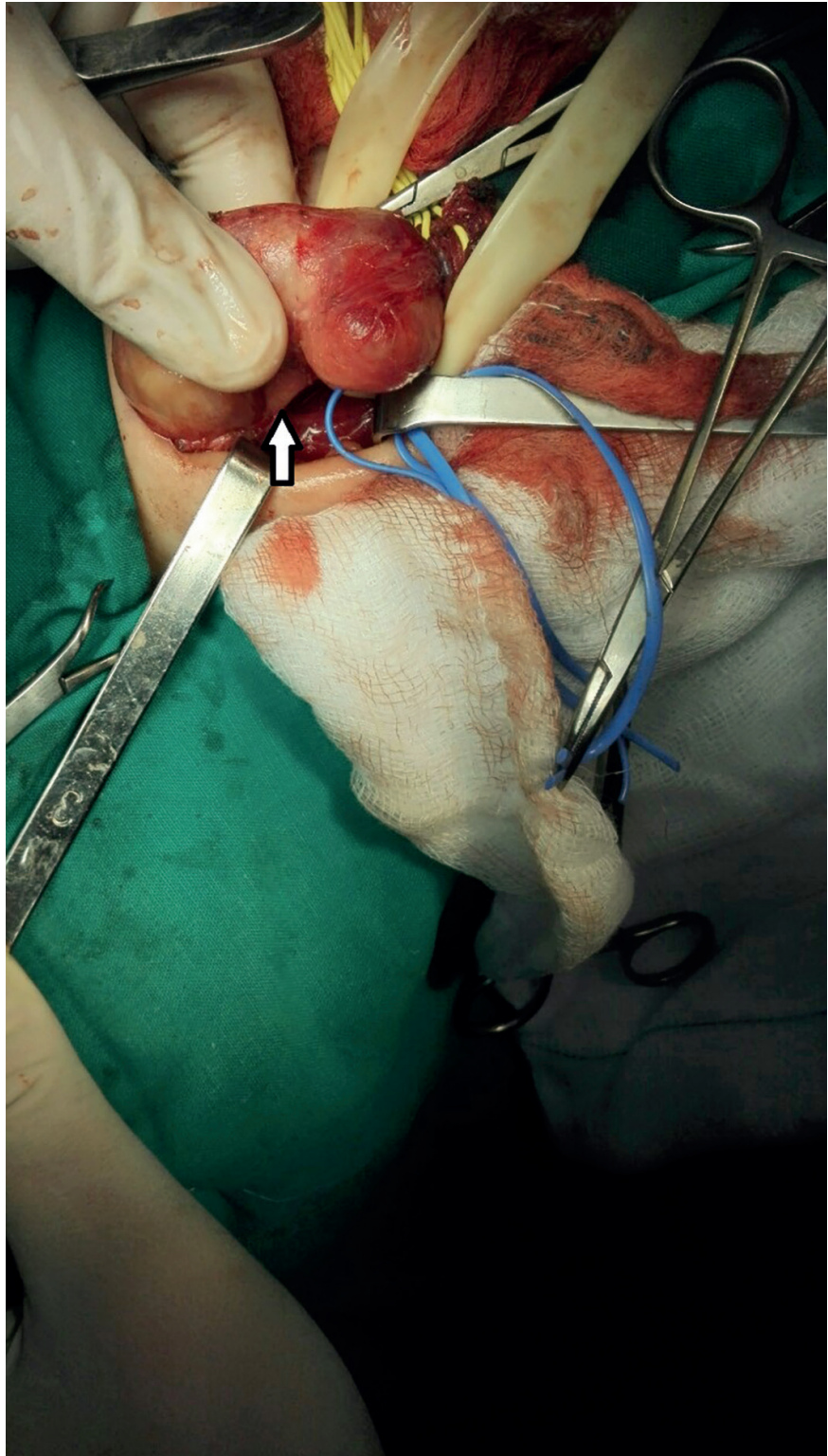

Fig. 5 The mass was bluntly dissected and completely removed, without being opened. Notice the close anatomic relationship with common carotid artery (white arrow).

Thymic cysts may be congenital; in such a case, they can be practically found anywhere along the thymo-pharyngeal duct (9). In their retrospective analysis, Bang et al. demonstrated that only 1 out of 16 cases examined in total corresponded to ectopic thymus located at the supraclavicular area (10). Rarely, thymic remnants are detected in atypical sites, such as the retropharynx (16). Alternatively, a thymic cyst may be acquired, developing after chemotherapy or thoracotomy, or even associated with thymic tumours distorting and compressing normal thymic tissue $(17,18)$. Overall, up to $70 \%$ of cervical thymic cysts are left-sided, while there is a slight predominance among males $(19,20)$.

The thymo-pharyngeal duct guides thymus descent towards the mediastinum $(1,2)$. Normally, the upper part of this canal regresses thereafter; any failure of its closure can lead to the formation of a congenital unilocular cervical thymic cyst. Degeneration of Hassall's corpuscles within remnants of ectopic thymic tissue can lead to genesis of

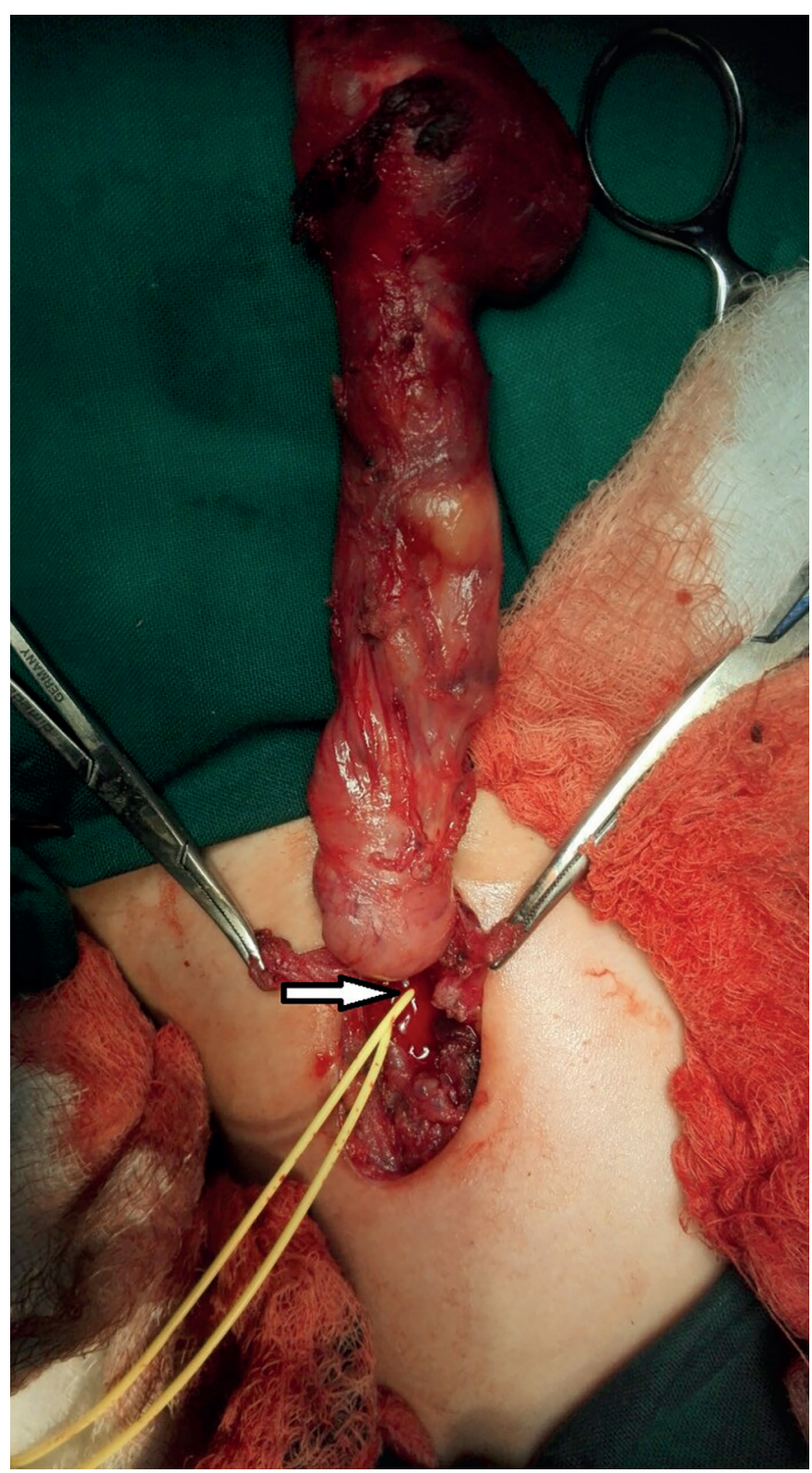

Fig. 6 The lower end of the cystic mass was adjacent to the upper pole of the left thyroid lobe (white arrow).

an acquired multilocular thymic cyst, as in our case. Thus, two main theories concerning cervical thymic cyst pathogenesis have been proposed by Speer; first, the persistence of the thymo-pharyngeal duct and second, the degeneration of Hassall's corpuscles $(21,22)$.

The size of a thymic cyst varies from 1 to $26 \mathrm{~cm}$ while in our case it was $14.5 \mathrm{~cm}$ (23). Most thymic cysts are asymptomatic, while in $6-13 \%$ of all cases, affected patient may present with stridor - as in our case -, dysphonia, dysphagia or hoarseness $(9,21,22,24)$. Thymic cyst may be complicated with infection or hemorrhage during its course, thus presenting in an acute manner (16). Recurrent upper respiratory tract infections and fluctuation in size are also commonly reported (25). Eifinger et al. describe a case of a ruptured thymic cyst in a neonate in the context of hemorrhage, leading to acute respiratory distress (26).

Valsalva maneuver may produce an apparent enlargement of the mass either due to the venous engorgement or via the increased intrathoracic pressure. Physical exami- 


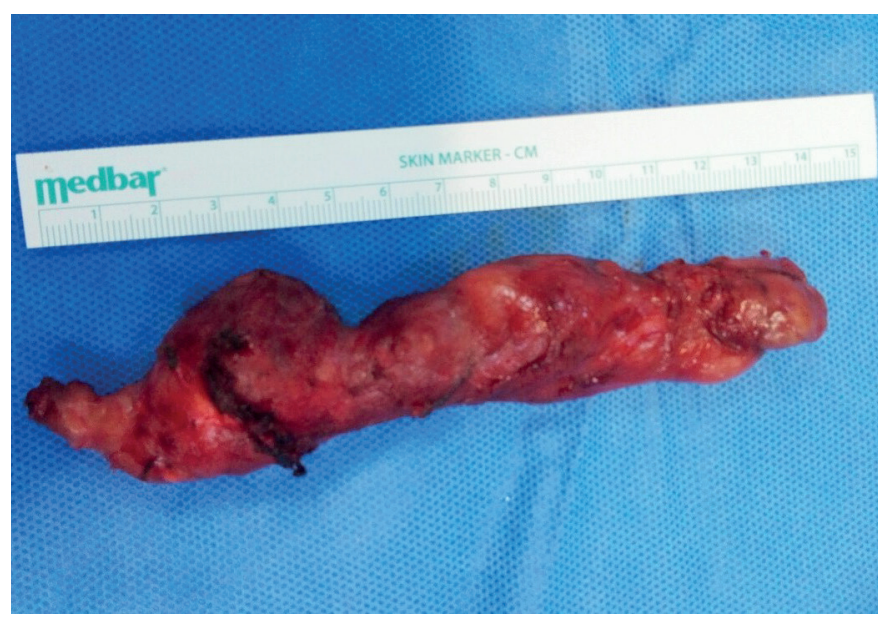

Fig. 7 Macroscopic view of the excised mass.

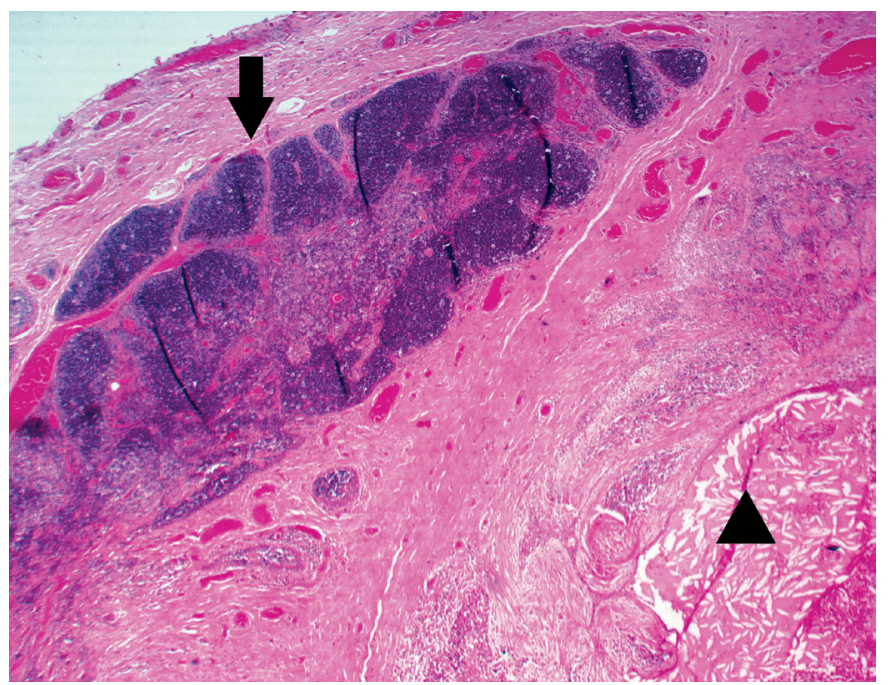

Fig. 8 Thymus gland cyst - Cystic area (arrowhead) adjacent to thymus parenchyma (arrow) [haematoxylin and eosin stain (H-E), $25 \times]$.

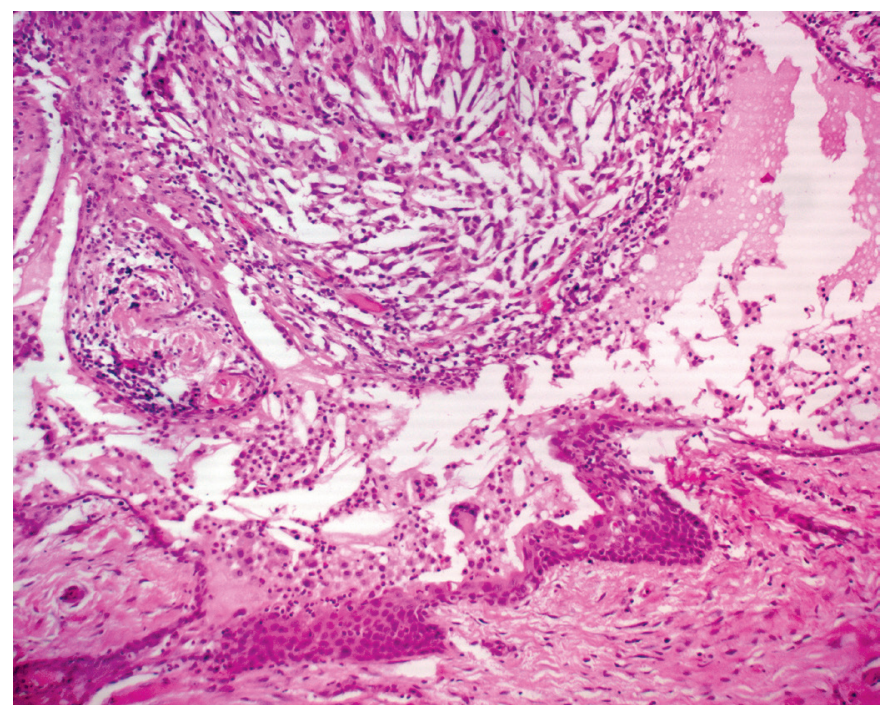

Fig. 9 Cyst filled with tissue detritus and cholesterol crystals, lined internally by stratified epithelium (H-E, 100x). nation should also focus on differential diagnosis between a cystic ectopic thymic remnant and a branchial cyst; in the latter case, a branchial cyst does not enlarge with Valsalva maneuver (27). Valsalva maneuver does not induce enlargement of the mass, when it does not extend to the mediastinum, as in our case. Mediastinal extension is seen in almost half of the cases of cervical thymic cysts (22).

Plain chest radiograph will reveal a normal thymic shadow in the mediastinum in children aged less than 2 years; thus, presence of mediastinal shadow beyond this age is suggestive of the mediastinal extension of a cervical thymic cyst (28). Performance of Doppler ultrasound enables the clinician to identify certain cyst characteristics, such as the presence of septae, the hypo- or anechoic content and its relationship with the adjacent anatomic structures (29). Occasionally, internal septations or mural calcifications can also be seen; however, cyst's content may be proteinaceous or hemorrhagic, thus creating a diagnostic dilemma (17). In such cases, magnetic resonance imaging (MRI) appears more accurate in distinguishing a thymic cyst from a solid thymic lesion (29). Therefore, MRI might be helpful, when evaluating a child with a solid cervical mass, in order to compare the derived imaging characteristics to those corresponding to an orthotopic mediastinal thymus (16).

In less than $15 \%$ of cervical thymic remnants diagnosis is made preoperatively $(30,31)$. In their retrospective analysis, Statham et al. managed 16 children with cervical thymic remnants, 3 solid and 13 cystic (27). Preoperative imaging techniques were not diagnostic in those patients having a solid thymic cyst, while they established the correct diagnosis in one fourth of those patients featuring a cystic thymic mass (27).

Thus, physical examination, preoperative imaging and intraoperative findings play a crucial role in the diagnosis of a cervical thymic cyst; however, diagnosis is usually established after histopathological examination $(7,22,24)$.

A thymic cyst usually contains ectodermal derivatives, with its epithelium ranging from squamous to cuboidal or columnar. It also contains endodermal derivatives, for example thymic and parathyroid tissue (7). Presence of thymic tissue, lymphoid tissue, and Hassall's corpuscles within the cystic mass constitute pathognomonic findings $(7,25,29)$.

Respiratory distress post resection of a thymic remnant is a known complication of cervical thymic masses. Presence of tracheal compression by the mass preoperatively is an important guide for postoperative respiratory care (27). In our case we reported a mild stridor that restored within a few minutes, without any signs of acute respiratory distress. Patients with respiratory distress symptoms during the postoperative period may be managed with emergent endotracheal intubation; however, no long-term sequelae is reported in most cases (25).

No immunological deficiency after removal of a thymic cyst is expected; this can be simply explained by the fact that it does not contain functional thymic tissue $(19,27)$. Although being extremely rare during childhood, complications in the presence of a thymic cyst that should be taken into account are thymic carcinoma and myasthenia gravis development $(9,19)$. 


\section{CONCLUSIONS}

1. Cervical thymic remnants represent a group of neck masses that pediatricians and pediatric surgeons should always consider in the differential diagnosis of both cystic and solid neck mass.

2. Most cervical thymic cysts are found in the lower third of the anterior neck, extending deep into the sternocleidomastoid muscle, with close anatomic relationship with the composites of the carotid sheath.

3. After ruling out the possibility of a subject immunologic deficit, elective surgical excision of the mass is recommended. No severe complications are described in the vast majority of cases.

\section{REFERENCES}

1. Nishino M, Ashiku SK, Kocher ON, Thurer RL, Boiselle PM, Hatabu H. The thymus: a comprehensive review. Radio Graphics 2006; $26(2)$ : $335-48$.

2. Lele SM, Lele MS, Anderson VM. The thymus in infancy and childhood: embryologic, anatomic, and pathologic considerations. Chest Surg Clin N Am 2001; 11(2): 233-53.

3. Ackman JB, Kovacina B, Carter BW, et al. Sex difference in normal thymic appearance in adults 20-30 years of age. Radiology 2013; 268(1): 245-53.

4. Goldstein AJ, Oliva I, Honarpisheh H, Rubinowitz A. A tour of the thymus: a review of thymic lesions with radiologic and pathologic correlation. Can Assoc Radiol J 2015; 66(1): 5-15.

5. Nasseri F, Eftekhari E. Clinical and radiologic review of the normal and abnormal thymus: pearls and pitfalls. Radiographics 2010; 30(2): 413-28.

6. Betti M, Hoseini NH, Martin A, Buccoliero A, Messineo A, Ghionzoli M. Cervical thymic cyst in childhood: a case report. Fetal Pediatr Pathol 2015; 34(1): 65-69.

7. Iqbal SM, Garg AK, Dubey A. Cervical thymic cyst-a case report. Indian J Otolaryngol Head Neck Surg 2005; 57(1): 67-68.

8. Hsieh YY, Hsueh S, Hsueh C, et al. Pathological analysis of congenital cervical cysts in children: 20 years of experience at Chang Gung memorial hospital. Chang Gung Medical Journal 2003; 26(2): 107-13.

9. Tandon A, Tandon R, Chandrashekhar M, Das P, Bansal B, Bhatia N. Cervical ectopic thymic cyst: a rare preoperative diagnosis. BMJ Case Rep 2011; 2011: pii: bcr052011425010.

10. Bang MH, Shin J, Lee KS, Kang MJ. Intrathyroidal ectopic thymus in children: A benign lesion. Medicine (Baltimore) 2018; 97(14): e0282.

11. Kabaalioğlu A, Öztek MA, Kesimal U, Ceken K, Durmaz E, Apaydın A. Intrathyroidal ectopic thymus in children: a sonographic survey. Med Ultrason 2017; 19(2): 179-84.
12. Avula S, Daneman A, Navarro OM, Moineddin R, Urbach S, Daneman D. Incidental thyroid abnormalities identified on neck US for non-thyroid disorders. Pediatr Radiol 2010; 40(11): 1774-80.

13. Kim HG, Kim MJ, Lee MJ. Sonographic appearance of intrathyroid ectopic thymus in children. J Clin Ultrasound 2012; 40(5): 266-71.

14. Millman B, Pransky S, Castillo J 3rd, Zipfel TE, Wood WE. Cervical thymic anomalies. Int J Pediatr Otorhinolaryngol 1999; 47(1): 29-39.

15. Prasad KK, Gupta RK, Jain M, Kar DK, Agarwal G. Cervical thymic cyst: report of a case and review of the literature. Indian J Pathol Microbiol 2001; 44(4): 483-5.

16. LaPlante JK, Pierson NS, Hedlund GL. Common pediatric head and neck congenital/developmental anomalies. Radiol Clin North Am 2015; 53(1): 181-96.

17. Strollo DC, Rosado-de-Christenson ML. Tumors of the thymus. J Thorac Imaging 1999; 14(3): 152-71.

18. Kumar V, Abbas A, Fausto N. Robbins and Cotran: Pathologic Basis of Disease. Philadelphia, PA: Elsevier Saunders; 2005.

19. Oztürk H, Karnak I, Deveci S, Sürer I, Cetinkursun S. Multilocular cervical thymic cyst: an unusual neck mass in children. Int J Pediatr Otorhinolaryngol 2001; 61(3): 249-52.

20. Jaiswal AA, Garg AK, Ravindranath M, Mohanty MK. Multi loculated cervical thymic cyst - case report with review of literature. Egypt J Ear Nose Throat Allied Sci 2014; 15(2): 129-33.

21. Hazmiri FE, Nachite F, Skandour D, Raji A, El Ganouni NCI, Rais H. Lateral cervical thymic cyst in a child: a case report. BMC Res Notes 2018; 11(1): 85.

22. Shenoy V, Kamath MP, Hegde MC, Rao Aroor R, Maller VV. Cervical thymic cyst: a rare differential diagnosis in lateral neck swelling. Case Rep Otolaryngol 2013; 2013: 350502

23. Nguyen Q, deTar M, Wells W, Crockett D. Cervical thymic cyst: case reports and review of the literature. Laryngoscope 1996; 106 (3 Pt 1): 247-52.

24. Srivalli M, Qaiyum HA, Srinivas Moorthy PN, Srikanth K. A case report of cervical thymic cyst and review of literature. Indian J Otolaryngol Head Neck Surg 2011; 63(1): 93-5.

25. Wagner CW, Vinocur CD, Weintraub WH, Golladay ES. Respiratory complications in cervical thymic cysts. J Pediatr Surg 1988; 23(7): 657-60.

26. Eifinger F, Hekmat K, Huenseler C, Vierzig A, Roth B. Life-threatening bleeding from thymic cysts in a newborn. Arch Dis Child Fetal Neonatal Ed 2007; 92 (5): F371.

27. Statham MM, Mehta D, Willging JP. Cervical thymic remnants in children. Int J Pediatr Otorhinolaryngol 2008; 72(12): 1807-13.

28. Ballal HS, Mahale A, Hegde V, Shetty R, Bhavikatti M. Cervical thymic cyst. Indian J Radiol Imaging 1999; 9: 187-9.

29. Tomiyama N, Honda O, Tsubamoto M, et al. Anterior mediastinal tumors: diagnostic accuracy of CT and MRI. Eur J Radiol 2009; 69(2): 280-8.

30. Tovi F, Mares AJ. The aberrant cervical thymus. Embryology, Pathology, and clinical implications. Am J Surg 1978; 136(5): 631-7.

31. Tunkel DE, Erozan YS, Weir EG. Ectopic cervical thymic tissue: diagnosis by fine needle aspiration. Arch Pathol Lab Med 2001; 125(2): 278-81. 